\title{
Análisis del discurso de odio sobre la islamofobia en Twitter y su repercusión social en el caso de la campańa "Quítale las etiquetas al velo»
}

\author{
Rocío Zamora Medina \\ Pilar Garrido Clemente \\ Jorge Sánchez Martínez \\ Universidad de Murcia \\ rzamoramedina@um.es \\ pilargarrido@um.es \\ jorge.s.m@um.es
}

Fecha de presentación: diciembre de 2020

Fecha de aceptación: abril de 2021

Fecha de publicación: diciembre de 2021

Cita recomendada: Zamora MEdina, R.; GARRIDO Clemente, P. y SÁNCHEZ MARTíneZ, J. (2021). "Análisis del discurso de odio sobre la islamofobia en Twitter y su repercusión social en el caso de la campaña "Quítale las etiquetas al velo"». Anàlisi: Quaderns de Comunicació i Cultura, 65, 1-19. DOI: <https://doi.org/10.5565/rev/analisi.3383>

\section{Resumen}

En el nuevo ecosistema mediático, las redes sociales se convierten en espacios clave para canalizar creencias políticas de odio, ideologías y acciones. Dada la escasez de trabajos empíricos en España sobre el discurso islamófobo en las redes sociales, esta investigación ofrece un análisis cuantitativo riguroso de la conversación social en Twitter generada a raíz de la campaña lanzada en 2019 bajo el título "Quítale las etiquetas al velo», con la que la Fundación Al Fanar para el Conocimiento Árabe, con apoyo de Twitter, pretendió desarticular las visiones estereotipadas de la mujer musulmana. Se llevó a cabo un análisis cuantitativo del impacto generado por el tuit principal de la campaña y la emisión de los dos hilos de información posteriores. La muestra $(N=1.545)$ la conformaron cada uno de los comentarios registrados en el tuit inicial de la campańa $(N=747)$ y en los dos hilos posteriores $(N=603$ y $N=195)$. Se intenta averiguar quién participó en la conversación, los temas, el tono y la principal acción que la definió. Posteriormente, se realizó un análisis crítico del discurso de los cinco tuits más determinantes en cada una de las acciones. Los resultados demostraron una superioridad occidental que califica al islam de manera despectiva. La interacción social entre los participantes fue escasa y se detectaron acciones islamófobas coordinadas de tipo ideológico en los contenidos promocionados por Twitter.

Palabras clave: islamofobia; Twitter; velo; islam; discurso de odio 
Resum. Anàlisi del discurs d'odi sobre la islamofobia a Twitter i la seva repercussió social en el cas de la campanya "Treu-li les etiquetes al vel"

En el nou ecosistema mediàtic, les xarxes socials es converteixen en espais clau per canalitzar creences polítiques d'odi, ideologies i accions. Atesa l'escassetat de treballs empírics a Espanya sobre el discurs islamòfob a les xarxes socials, aquesta investigació ofereix una anàlisi quantitativa rigorosa de la conversa social a Twitter generada arran de la campanya llançada el 2019 sota el títol «Treu-li les etiquetes al vel», amb la qual la Fundació Al Fanar per al Coneixement Àrab, amb suport de Twitter, va pretendre desarticular les visions estereotipades de la dona musulmana. Es va dur a terme una anàlisi quantitativa de l'impacte generat pel tuit principal de la campanya i l'emissió dels dos fils d'informació posteriors. La mostra $(N=1.545)$ la van conformar cada un dels comentaris registrats en el tuit inicial de la campanya $(N=747)$ i en els dos fils posteriors $(N=603$ i $N=195)$. S'intenta esbrinar qui va participar en la conversa, els temes, el to i l'acció principal que la va definir. Posteriorment, es va fer una anàlisi crítica del discurs dels cinc tuits més determinants en cadascuna de les accions. Els resultats van demostrar una superioritat occidental que qualifica l'islam de manera despectiva. La interacció social entre els participants va ser escassa i es van detectar accions islamòfobes coordinades de tipus ideològic en els continguts promocionats per Twitter.

Paraules clau: islamofòbia; Twitter; vel; islam; discurs d'odi

Abstract. Analysis of hate speech involving Islamophobia on Twitter and its social repercussion in the case of the campaign "Remove the labels from the veil"

In the new media ecosystem, social networks become a critical space for channelling hatefilled political beliefs, ideologies and actions. Given the scarcity of empirical studies related to Islamophobic discourse on social networks in Spain, this study offers a rigorous quantitative analysis of social conversation on Twitter generated as a result of the "Remove the labels from the veil" campaign launched in 2019 by Al Fanar Foundation for Arab Knowledge, with the support of Twitter, in order to dismantle stereotypical views of Muslim women. A quantitative analysis of the impact generated by the campaign's main tweet and the publishing of two subsequent information threads was carried out. The sample $(\mathrm{N}=1,545)$ was made up of each comment registered in the initial tweet of the campaign $(N=747)$ and in the two subsequent threads $(N=603$ and $N=195)$. The aim was to establish who participated in the conversation, and the main topics, tone and actions that defined it. Subsequently, a critical analysis of the discourse of the five most determining tweets in each of the actions was carried out. Results showed a Western superiority that labels Islam in a derogatory way. Social interaction among participants was limited, and coordinated ideological Islamophobic actions were detected in content promoted by Twitter.

Keywords: Islamophobia; Twitter; veil; Islam; hate speech

\section{Introducción}

En el ecosistema de los nuevos medios, las redes sociales se convierten en un "vehículo para canalizar creencias, ideologías y acciones políticas de odio" (Kopytowska, 2017). La presencia de contenidos emocionales —en especial, los más negativos - cuando son compartidos a través de las redes sociales 
«tienen resonancia en quien los publica y en quienes los leen o ven, produciéndose una respuesta emocional con alta concentración de sentimiento» (Peris-Hernández, Maganto-Mateo y Kortabarria-Kortazar, 2018). Tal como han demostrado algunos estudios recientes (Arcila, Blanco-Herrero y Valdez Apolo, 2020; Paz, Montero-Díaz y Moreno-Delgado, 2020; Chakraborti, Garland y Hardy, 2014; Miró, 2016), el odio es una de las emociones más recurrentes en el mundo virtual. De hecho, se ha acuñado la expresión ciberodio para referirse a las conductas de odio que son visibles a través de la red, lo que, en la mayoría de los casos, se concreta en propaganda de índole racista, xenófoba y violenta difundida por medio de sistemas digitales. Chakraborti et al. (2014: 24) lo definen como "cualquier acto digital de violencia, hostilidad e intimidación, dirigido a las personas debido a su identidad o la diferencia que se perciba».

Este trabajo ofrece un análisis de un caso vinculado con los discursos de odio en torno a la islamofobia. Considerados como un tipo de discurso de odio discriminatorio sobre la población musulmana o el islam (Cheng, 2015) por incitar a la discriminación o a la violencia por motivos de odio racial, xenófobo, orientación sexual u otras formas de intolerancia (Gagliardone, 2015), la islamofobia se expresa a través de la violencia verbal que juzga al islam como incompatible con Occidente. Se trata de un objeto de estudio polémico, en la medida en que se genera tensión entre el derecho a la libertad de expresión y el respeto religioso (Campos, 2017).

Concretamente, la investigación se centra en la campaña "Quítale las etiquetas al velo», coordinada por la Fundación Al Fanar. Se trata del colectivo que despierta mayor interés por parte de los investigadores, debido a su posición de sujeto simbólico con el que se identifica la identidad musulmana (Garrido, 2013; Torres y Garrido, 2013). A partir de este hecho se organizó, en el año 2018 en Valencia, el Laboratorio de innovación social para combatir la islamofobia de género, donde se diseñó el contenido y la estrategia de esta campaña. La campaña quedaba sintetizada en el hashtag \#velosinetiquetas, con el que se ofrecía una visión plural y auténtica de las mujeres musulmanas, yendo más allá de los estereotipos. El vídeo se lanzó el 21 de septiembre de 2019, coincidiendo con el Día Europeo contra la Islamofobia. La campańa formaba parte del proyecto europeo Stop-Islamophobia y contó con el apoyo de Twitter for Good. El respaldo de Twitter permitió llegar a casi 8 millones de interacciones y 2,5 millones de visualizaciones del vídeo. Además, generó más de 3.500 «me gusta» y retuits, pero también más de 1.500 mensajes, la mayoría de ellos agresivos e islamófobos, que componen el corpus que analizamos.

Siguiendo las recomendaciones de la Guía para medios de comunicación "Islam, personas musulmanas y periodismo» (Rojo, 2020), se decidió que, ante aquellos mensajes ofensivos, pero no denunciables, la mejor estrategia es eludir un diálogo directo con sus emisores y no participar en conversaciones con hashtags islamófobos, que redundan en una mayor difusión de su mensaje ofensivo. Así, se creó contenido alternativo basado en hechos y datos para 
generar una dialéctica positiva que diera voz a cuentas y mensajes constructivos. Por este motivo se confeccionaron dos hilos informativos. El primero estaba compuesto por testimonios de mujeres musulmanas españolas compartiendo sus puntos de vista personales, mientras que el segundo hilo compilaba citas académicas sobre la cuestión. Los comentarios de ambos hilos han sido analizados en el presente estudio.

Partimos de la hipótesis de que los discursos sociales que se generan en las redes sociales en torno a la islamofobia se caracterizan por la ausencia de diálogo entre los diferentes actores, así como por sus altas dosis de negatividad. El carácter especial de «hipermediación» de los tuits islamófobos favorece la difusión de actitudes islamófobas (Evolvi, 2019) y la polarización.

Si bien la reflexión sobre el discurso islamófobo ha generado diferentes discusiones académicas sobre la identidad y las minorías (Pérez de la Fuente, 2010), hasta el momento la bibliografía resulta escasa, por lo que resultan necesarias más contribuciones innovadoras que actúen desde una perspectiva empírica, analítica e interdisciplinaria. Los pocos informes sobre la imagen musulmana en Internet en Espańa muestran que estos tienen una representación negativa y estigmatizada en los espacios en línea (Alcántara-Plá y Ruiz-Sánchez, 2017; Ruiz-Sánchez y Alcántara-Plá, 2018). Además, predomina una narrativa relacionada con la radicalización y el terrorismo, siendo la estigmatización el primer paso en la escalada hacia el discurso de odio y los delitos de odio. La información sobre la comunidad musulmana es, además, objeto de numerosos bulos y noticias falsas, hasta el punto de que, como algunos estudios recientes han apuntado, «la interrelación que se establece entre bulos sobre migración y religión musulmana es quizá la más evidente y un desconocimiento de la propia diversidad interna que ayuda a fomentar ciertos estereotipos» (Magallón, 2020).

El aumento de la intolerancia contra la población musulmana en Europa ha sido reportado en los últimos años por diferentes instituciones y organizaciones, entre los que destacamos el trabajo sobre discriminación social incluido en el Eurobarómetro Especial sobre Discriminación en la UE, de 2015. Además, según el marco jurídico europeo sobre el discurso del odio, la blasfemia y su interacción con la libertad de expresión, el discurso de odio está creciendo en Europa en general siendo el discurso de odio musulmán en línea el más frecuente (ECRI, 2016).

La abrumadora existencia del discurso del odio en línea como un fenómeno extremadamente dañino ha llevado a la creación de campañas masivas europeas, como el movimiento No Hate Speech, del Consejo de Europa, que pretenden servir de respuesta ante el incremento del discurso de odio en los diferentes países europeos. Asimismo, son numerosas las iniciativas europeas centradas en ofrecer recursos para afrontar el discurso del odio, como la que publicó The Media Diversity Institute (MDI) bajo el título Stopping Hate: How to Counter Hate Speech on Twitter? (Iganski y Sweiry, 2018).

En España, concretamente, la situación no es menos preocupante. Según los datos del Observatorio de la Islamofobia en los Medios (IEMed, 2018), el 
$60 \%$ de los artículos publicados en la prensa espańola tiene connotaciones islamófobas. La situación se agrava en los artículos de opinión, con un 72\% de las piezas con tintes islamófobos.

En definitiva, tal y como apuntan estos trabajos previos, aunque la realidad social de los musulmanes en España es diversa (incluyendo a españoles, inmigrantes, turistas, refugiados, etc.), partimos de la hipótesis de que el discurso específico sobre la comunidad musulmana en Internet es superficial y tendencioso, y está claramente estigmatizado. Incluso cuando se mencionan desde una perspectiva histórica, se representan casi exclusivamente como invasores militares (un "ellos» como enemigos históricos en nuestro territorio). En los pocos casos que se enmarcan como víctimas de injusticias relacionadas con la islamofobia también se construyen como un "ellos» opuestos al «nosotros» (Alcántara-Plá y Ruiz-Sánchez, 2017). Nuestro objetivo será comprobar hasta qué punto la conversación en Twitter generada a raíz de la campaña lanzada favoreció el diálogo social en aras de lograr un enmarcado más constructivo de este colectivo o bien solo sirvió para perpetuar la conflictividad y la polémica sustentada en las diferencias ideológicas de los usuarios.

\section{Metodología}

El diseño metodológico consistió en un análisis de contenido de todos los comentarios publicados a raíz del tuit central en el que se difundió la campaña \#velosinetiquetas. Este tuit se emitió el día 21 de septiembre de 2019, coincidiendo con el Día Europeo contra la Islamofobia, e incluía un texto "¿Las telas te confunden? ¡A Aparta tus prejuicios y ve personas, no cosas! ¡Dentro vídeo! \#velosinetiquetas», e incluía el vídeo Velo sin etiquetas. A raíz de la emisión de este tuit se registraron 747 comentarios. En total, la muestra $(N=1.545)$ la conformaron cada uno de los comentarios registrados, que se tomaron como unidad de análisis, incluyendo aquellos derivados del primer tuit inicial de la campańa $(N=747)$, así como tras los dos hilos posteriores $(N=603$ y $N=195)$.

Se establecieron varios grupos de categorías descriptivas, que incluyeron datos métricos de cada comentario (número de retuits, «me gusta»), optimización de recursos propios de Twitter (hashtags, emoticonos, menciones, recursos visuales), así como variables claves para conocer el contenido de cada comentario, en relación con el tema principal, la acción principal o el tono del comentario y la fuente o autor del mismo.

El proceso de codificación fue realizado manualmente por los autores de la investigación a través del análisis de una muestra previa de 50 tuits de cada uno de los hilos. Tras dos rondas de codificación se obtuvo un nivel de acuerdo entre codificadores del 91,3\%. Posteriormente, se utilizó el programa informático SPSS versión 20 para proceder al análisis estadístico de las frecuencias, así como la elaboración de tablas de contingencia.

En líneas generales, la primera de las acciones de la campaña (A1) obtuvo una resonancia mayor que el resto, no solo por contar con mayor número de 
comentarios, sino también porque alcanzó casi el millón de interacciones y un total de 507 retuits y 699 "me gusta». En el caso del hilo de testimonios (A2), el número de retuits fue de 688 y el de "me gusta», 1,3 mil. Finalmente, el hilo académico (A3) obtuvo 541 retuits y 1,4 mil. de «me gusta».

Además, para conocer el tipo de usuario que los publican se realizó un análisis de contenido de los comentarios derivados de una cuarta acción (A4) correspondiente con el lanzamiento en Twitter de un HT convertido en trending topic (en adelante, TT) en el contexto de la presentación de una guía para periodistas realizada por la Fundación Al Fanar con el objetivo de ofrecer claves para escribir de forma inclusiva sobre el islam y las personas musulmanas. Twitter convirtió el HT \#VeloSinEtiquetas en un TT promocionado. En esta acción participaron un total de 4.264 usuarios y se realizaron 5.940 retuits. Para el tratamiento de estos datos se utilizó la herramienta T-Hoarder Kit (Congosto, 2016) y el programa Gephi para su representación.

El análisis cuantitativo se completó con un análisis cualitativo posterior sustentado en el análisis crítico del discurso (ACD) como técnica idónea para desvelar el significado contenido a partir de la detección de las relaciones sociales que se establecen y se potencian a través del uso del lenguaje, considerando el discurso como la reproducción de la dominación o el abuso de poder (Van Dijk, 2002). El objetivo era identificar el uso de generalizaciones, visiones estereotipadas, vínculos y patrones interpretativos, así como el tono de los mismos, que configuró el discurso cualitativo en relación con la islamofobia. Para ello se tomó como base los cinco tuits más determinantes en cada una de las cuatro acciones analizadas, seleccionados a partir del mayor número de retuits, y que permitió profundizar en las cuestiones de tipo lingüístico y semántico del discurso.

\section{Resultados}

\subsection{Protagonismo de la superioridad occidental como tema de discusión}

En líneas generales, los comentarios incluidos en nuestra muestra versaron mayoritariamente sobre la superioridad occidental como tema principal (un $42,1 \%$ ), seguido de otros temas como el velo como imposición (un 16,3\%), el islam como religión machista (un 13,5\%) y el velo como símbolo machista (un 12,7\%). Este protagonismo de la superioridad occidental, entendida como la existencia implícita de un choque de civilizaciones en el que Occidente se reconoce como superior, y por lo tanto es quien impone las reglas, estuvo menos presente en la segunda acción de la campańa, que se corresponde con el hilo de testimonios (con un 38,5\%), donde el velo como imposición fue también tema destacado (un 25\%). Sin embargo, en la tercera acción, el hilo académico aumentó considerablemente la idea de la superioridad occidental, alcanzando el $61,5 \%$ de los casos. 
Gráfico 1. Distribución de temas según las diferentes acciones de la campaña

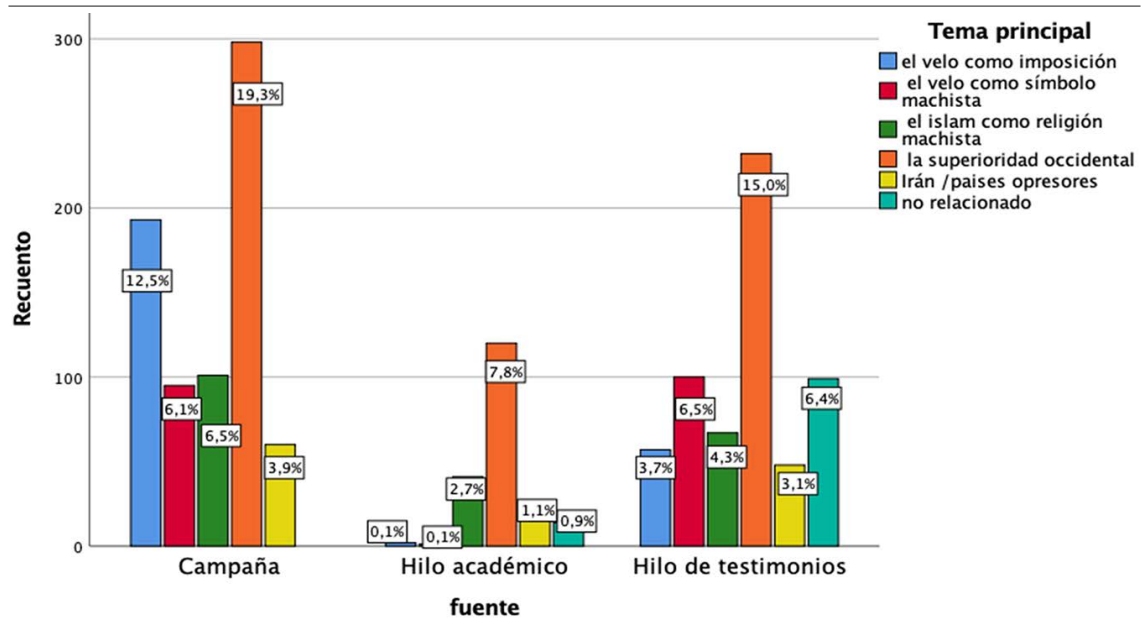

Fuente: elaboración propia.

\subsection{Predominio del tono negativo e intencionalidad critica}

En general, el discurso sobre la islamofobia que caracterizó a esta campaña fue notablemente negativo (un $71,2 \%$ ). Esta pauta se mantuvo en todas las acciones en líneas similares, de tal modo que tres de cada cuatro comentarios se caracterizaron por un tono negativo. El porcentaje mayor de comentarios positivos se detectó en los momentos iniciales, tras la primera acción (un 20,2\%).

A pesar de que el tono negativo caracterizó los comentarios de todos los temas, este se acentuó en los comentarios sobre el islam como religión

Tabla 1. Tema principal en función del tono del comentario

\begin{tabular}{lcccc}
\hline & Positivo & Negativo & Neutro & Total \\
\hline El velo como imposición & 66 & 169 & 17 & 252 \\
& $26,2 \%$ & $67,1 \%$ & $6,7 \%$ & $100 \%$ \\
El velo como símbolo machista & 29 & 163 & 4 & 196 \\
& $14,8 \%$ & $83,2 \%$ & $2 \%$ & $100 \%$ \\
El islam como religión machista & 18 & 185 & 6 & 209 \\
& $8,6 \%$ & $88,5 \%$ & $2,9 \%$ & $100 \%$ \\
Superioridad occidental & 124 & 469 & 57 & 650 \\
& $19,1 \%$ & $72,2 \%$ & $8,8 \%$ & $0 \%$ \\
Irán / países opresores & 8 & 109 & 8 & 125 \\
& $6,4 \%$ & $87,2 \%$ & $6,4 \%$ & $100 \%$ \\
No relacionado & 0 & 5 & 108 & 113 \\
& $0 \%$ & $4,4 \%$ & $95,6 \%$ & $100 \%$ \\
Total & 245 & 1100 & 200 & 1545 \\
& $100 \%$ & $100 \%$ & $100 \%$ & $100 \%$ \\
\hline
\end{tabular}

Fuente: elaboración propia. 
machista, así como aquellos sobre Irán y otros países opresores. Por el contrario, los escasos comentarios positivos fueron detectados entre los comentarios referidos al velo como imposición.

Se comprobó además que quienes participaron en la conversación social lo hicieron con un tono mayoritariamente crítico (un 45,1\%), con un porcentaje de insultos detectados notable (un 33,3\%), sobre todo a raíz de la primera acción. Por el contrario, destaca la casi inexistencia de la intención de difundir información (solo un 1,7\%). Dicho patrón se repite en las tres acciones de campaña con ciertos matices.

Si bien la acción crítica fue mayoritaria en los comentarios, esta se acentuó cuando los comentarios versaban sobre Irán / países opresores (un 63,2\%). La mayor presencia de insultos se detectó en los comentarios que abordaban la cuestión del islam como religión machista (un 39,7\%), así como la superioridad occidental (un 39,8\%).

\subsection{Escaso nivel de interacción social}

Uno de los recursos más utilizados en Twitter para favorecer la conversación social es el uso de hashtags. En este caso, el uso de hashtags resultó prácticamente anecdótico (un 99,2\% de los comentarios no incluían ninguno). Este patrón se repitió en todas las acciones de la campaña, salvo en el primer momento, que sí se utilizó en algún caso el hashtag oficial de la campaña. En las acciones posteriores aparecieron algunos otros más vinculados con el feminismo y otras cuestiones de tipo ideológico, tal y como se puede comprobar en el hilo académico — con los hashtags \#Islam2Gracias, \#StopIslam, \#MasFeminismoPorFa, \#stopislam, \#islamessumision-y el hilo de testimonios -con los hashtags \#2Cuela, \#Bullshit, \#Espana, \#feminismo, \#FreeFromHijab, \#Heteropatriarcadoislamista, \#VoxExtremaNecesidad.

Respecto al uso de menciones, al igual que ocurrió con los hashtags, los comentarios prácticamente no hicieron uso de este recurso para la interacción social. Las escasas menciones se refirieron a @nesssrin_, en la primera acción, asícomo a @lmamofpeace, @Ntumultuaria, @TwitterEspana, en la segunda acción,y, finalmente,@Absolutexe, @TwitterEspana, @xiomirb, y@zkhilah, en la tercera acción.

En la misma línea, el uso de emoticonos como recurso expresivo de la conversación fue bastante escaso (un 94,3\%), una pauta que se repitió en todas las acciones de la campaña.

A pesar de la importancia cada vez mayor de los recursos audiovisuales en Twitter, la mayoría de los comentarios (un 91\%) no incluyeron ningún tipo de contenido visual, ni fotografía ni vídeo, y se limitaron al contenido textual. En este sentido, la ausencia de fotografías o vídeos fue el común denominador en todos los comentarios, con independencia del tema. Solo en el caso de los comentarios referidos a Irán / países opresores se utilizaron este tipo de recursos audiovisuales con mayor frecuencia que en el resto (un 24\%). 


\subsection{Se viralizan más los comentarios negativos y críticos, vinculados con temas polémicos}

El estudio de esta campańa no puede dejar de advertir el análisis del impacto real que tuvieron los mensajes difundidos a modo de comentarios de los usuarios de Twitter a partir del número de retuits. A raíz de la difusión del vídeo como primera acción de la campaña, hubo dos cuestiones - el islam como religión machista y la mención a Irán / países opresores- que fueron claramente más retuiteadas que el resto. Este patrón se modificó ligeramente en el caso de las dos acciones estratégicas posteriores, con un tema más que se coló en el hilo académico como fue la superioridad occidental, así como la importancia del velo como símbolo machista, que llegó a ser incluso más viralizado que la cuestión del islam como religión machista en el caso del hilo de testimonios.

Por lo que se refiere al tono de los mensajes, con independencia de la acción, aquellos tuits enmarcados en un tono negativo fueron también más retuiteados que los positivos o neutros. Del mismo modo se viralizaron los comentarios basados en la acción de criticar e insultar. Estos datos confirman el mayor potencial de viralidad que tiene la negatividad en esta red social. Esta circunstancia fue más evidente cuando el tuit versaba sobre Irán / países opresores, así como el velo como símbolo machista, cuyos tuits enmarcados negativamente obtuvieron un impacto mayor que en otros temas.

Gráfico 2. Media de retuits en función del tema y del tono principal

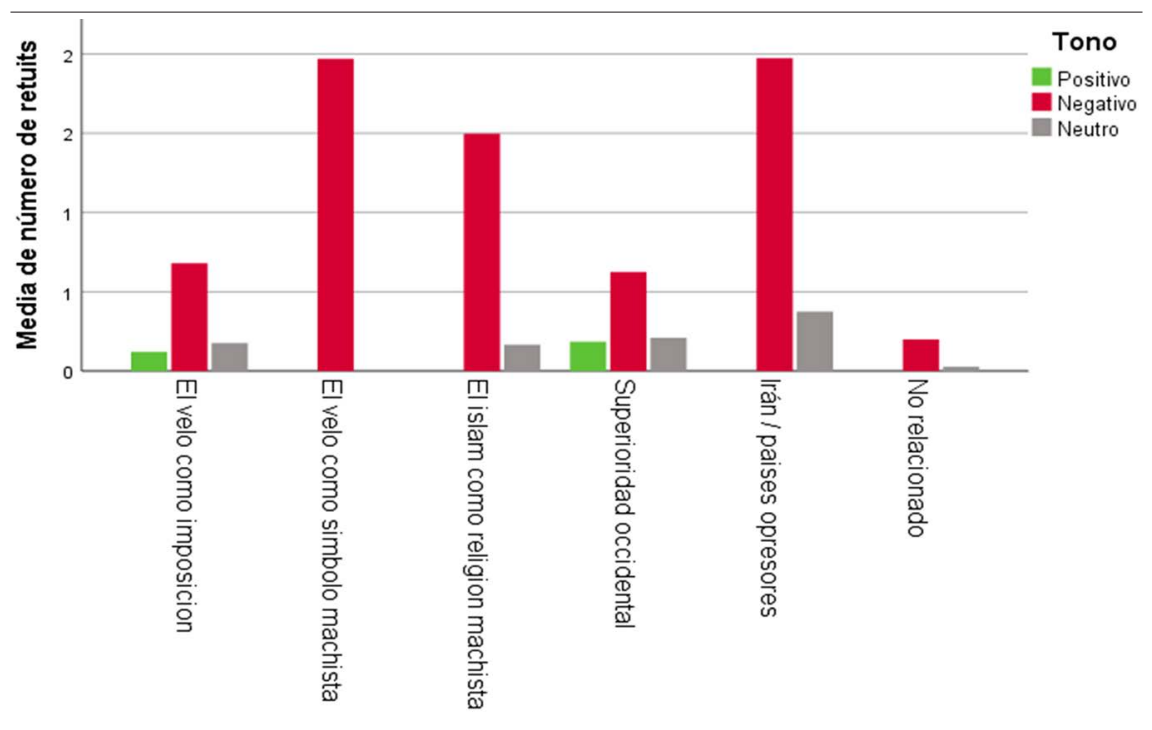

Fuente: elaboración propia. 
Finalmente, destacamos la importancia que tienen los contenidos audiovisuales en Twitter, en el sentido de que aquellos tuits que incluyeron imagen o vídeo obtuvieron considerablemente un número de retuits superior al resto. Este dato llegó a alcanzar la proporción de cuatro veces superior en el caso del hilo de testimonios y, por el contrario, no resultó tan determinante en el hilo académico.

\subsection{Aportaciones puntuales y discretas por parte de los usuarios que participan en la conversación social}

Otro de los objetivos de este estudio ha sido comprobar los usuarios que interactuaron durante las distintas acciones de la campaña. Para ello se tomó en consideración el agrupamiento de los usuarios en función del número de comentarios aportados por cada uno. Denominamos «usuarios puntuales» a los que hicieron únicamente una aportación; «usuarios frecuentes» a aquellos que hicieron más de un comentario pero menos de cinco, y «superusuarios» a aquellos que realizaron más de cinco aportaciones. En el cómputo global de los datos analizados figuran un total de un $86,1 \%$ de usuarios puntuales, un $11,1 \%$ de usuarios frecuentes y un $2,8 \%$ de superusuarios.

$\mathrm{Si}$ especificamos estos datos a partir de cada una de las tres acciones de la campaña, podemos ver posibles diferencias, sobre todo si se repiten los superusuarios entre una acción y otra. Así, en la primera acción de campaña se observó un 2,2\% de superusuarios, un 9,2\% de usuarios frecuentes y un $88,6 \%$ de usuarios puntuales. Por lo que se refiere a los comentarios del hilo académico, se identificaron cifras bastante similares, con un 2,5\% de superusuarios, un $11,2 \%$ de usuarios frecuentes y un $86,3 \%$ de usuarios puntuales. Respecto al hilo de testimonios encontramos una cifra algo superior de superusuarios (un 3,6\%) y de usuarios frecuentes (un 11,1\%), con un 85,3\% de usuarios puntuales.

\subsection{Mayor repercusión de los difusores de ideología en el TT promocionado}

La cuarta acción analizada tiene que ver con la conversación social en torno al HT promocionado \#VeloSinEtiquetas. El hecho de que fuera un TT promocionado supone notables diferencias. La más importante es que su repercusión es mucho mayor que la publicación de un tuit o un hilo de tuits que solo aparecen en la cronología de unos usuarios concretos. Esta circunstancia provoca que el número de comentarios sea mucho mayor que en el resto de las acciones. Por otra parte, el tipo de usuarios que participa también es diferente: todos los usuarios pueden ver ese TT en sus tendencias y tiene un mayor interés participar en esa conversación por su repercusión potencial.

Para analizar la difusión de los mensajes publicados con el HT \#VeloSinEtiquetas y las comunidades de tuiteros afines que se producen en torno a los discursos a favor y en contra de la campaña, nos centramos en un aspecto objetivo de la conversación: los retuits. Así, si analizamos los retuits que se 
han producido entre los distintos participantes de una conversación, podemos diferenciar comunidades de tuiteros afines y determinar qué usuarios han sido los más influyentes en la conversación.

El gráfico 3 representa los resultados de la conversación social generada en Gephi, donde los puntos representan las cuentas que participaron en el HT \#VeloSinEtiquetas y las flechas que unen los distintos puntos representan los retuits de un usuario a otro. Cuanto más grande es un punto y más flechas tiene, más difusión tuvieron sus publicaciones. Los retuits aproximan a los usuarios. Por tanto, los tuiteros que más se retuitean entre ellos se representarán más próximos y alejados de aquellos con los que no interaccionan. Así se crean las comunidades de tuiteros afines, cada una representada con un color.

Gráfico 3. Mapa en Gephi de la difusión de los mensajes publicados en \#VeloSinEtiquetas

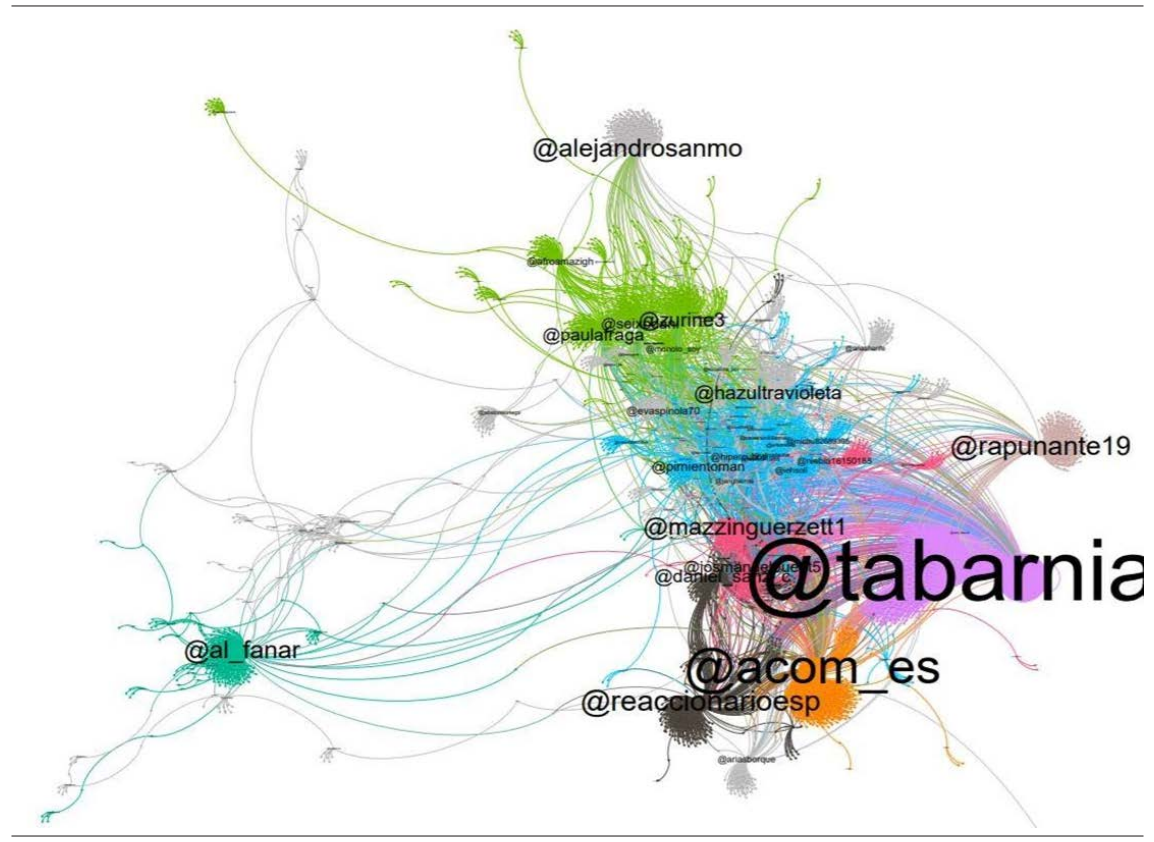

Fuente: elaboración propia.

En la imagen aparecen dos grandes grupos claramente diferenciados. A la izquierda, los que apoyan la campaña \#VeloSinEtiquetas, liderados por Al Fanar. A la derecha, los detractores, liderados por la cuenta Tabarnia. Como se puede comprobar, la difusión y el número de mensajes contra la campaña fue notablemente superior. Por lo que respecta a los usuarios que se manifestaron en contra de la campaña \#VeloSinEtiqueta, tal y como se aprecia en el gráfico 3, los que tuvieron más difusión fueron @tabarniabcn, @acom_es, @reaccionarioesp y @mazzinguerzett1. Todas estas cuentas se dedican netamente a la difusión de ideología marcadamente de derechas y con un discurso 
abiertamente islamófobo. Estos usuarios que más difusión han obtenido en la conversación social se dedican a llevar los debates políticos de actualidad hacia sus posiciones ideológicas con gran influencia, aprovechando la repercusión de estos mensajes.

Mención aparte merece la cuenta @acom_es, por ser una cuenta institucional y no personal destinada a fomentar las relaciones entre países difundiendo mensajes marcadamente islamófobos. Pertenece a la organización ACOM, Acción y Comunicación sobre Oriente Medio, que "promueve la relación entre España e Israel a través del trabajo conjunto con gobiernos, partidos políticos y sociedad civil».

Figura 1. Captura de la biografía de @acom_es

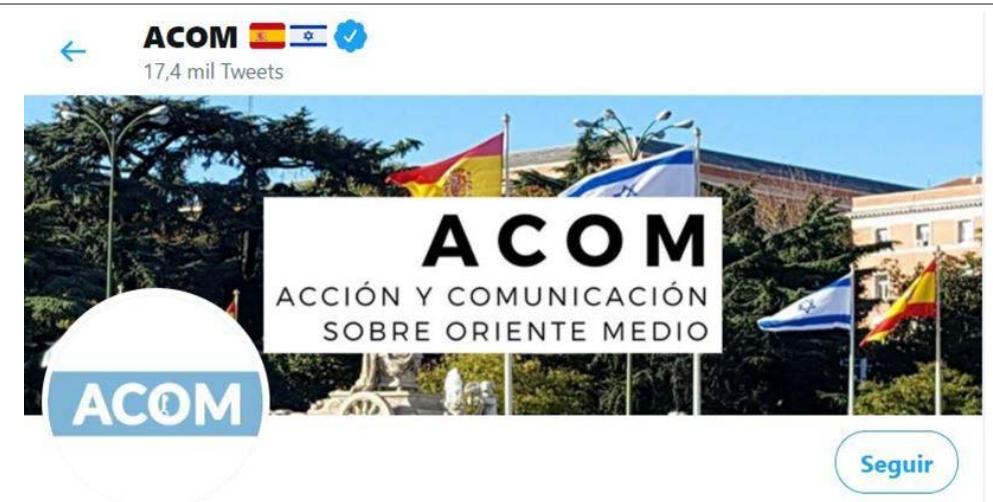

ACOM 프무

@ACOM_es

Organización independiente que promueve la relación entre España e Israel a través del trabajo conjunto con gobiernos, partidos políticos y sociedad civil.

(2) Spain $\mathcal{O}$ a-com.es : Se unió en abril de 2011

1.724 Siguiendo $\quad 14,1$ mil Seguidores

Tweets

Tweets y respuestas

Fotos y videos

Me gusta

Fuente: <https://twitter.com/ACOM_es>.

Otro aspecto destacado es que, aunque predominan los perfiles conservadores, hay una importante presencia de perfiles progresistas. La determinación de la ideología de las cuentas analizadas se ha realizado en base a las definiciones que los propios usuarios presentan en su perfil, de tal modo que perfilescomo@alejandrosanmo (que en la fecha de redacción de este artículo ha borrado su cuenta), @socialista_bcn (que ha cambiado su nombre por @FortunyEusebi) o @zurine3 son usuarios que se autodefinen como de izquierdas y participan en la conversación \#VeloSinEtiquetas difundiendo mensajes en contra del velo. Esta circunstancia demuestra que la islamofobia 
es una cuestión transversal a la cuestión ideológica y que, aunque se presenta con más intensidad entre los perfiles conservadores, también se detecta entre los progresistas.

Dentro de los argumentos más utilizados en los perfiles en contra del velo destaca la utilización de una noticia que cuenta la «condena a 55 años de cárcel de tres activistas iraníes por negarse a llevar velo» como una forma de asociar el uso del velo a la imposición y a los regímenes totalitarios. Esta argumentación se ha detectado entre perfiles tanto conservadores como progresistas.

En lo que se refiere a los usuarios que defienden la campaña \#VeloSinEtiquetas, estos se dividen en dos partes claramente diferenciadas. Por un lado, la comunidad tuitera en torno a Al Fanar, promotor de la campańa, y los perfiles que difundieron sus mensajes. Es, con diferencia, el perfil más retuiteado entre los que tuitearon a favor del HT. Por otro lado, encontramos una serie de usuarios que se posicionan a favor de la campaña y, además, entran a debatir con los detractores en el TT. Son en su mayoría mujeres y reciben una gran cantidad de mensajes de réplica. El tipo de conversación que se genera en este caso es similar al analizado en las otras acciones que se tratan en la investigación. Como muestra de estos usuarios tenemos a perfiles como @maryisattour, @laylaam20y@ibti_25.

\subsection{Discurso destructivo y generalizador basado en el desconocimiento del islam}

Para realizar este análisis cualitativo hemos extraído una muestra de los cinco tuits con más retuits de las tres acciones analizadas, así como de la cuarta acción relativa a las interacciones de los usuarios. El análisis indica de manera explícita y repetida que predominan los mensajes islamófobos, lo que generaliza una visión negativa del islam, incluso de la cultura derivada de la propia religión y sus tradiciones, que en la mayoría de los relatos se muestran como primitivas, irrespetuosas y sancionadoras. Esta visión va unida al desconocimiento de la religión y de la civilización islámicas, haciéndolas portadoras de ideas y mensajes anacrónicos y deformantes.

Otra cuestión determinante fue la generalización anuladora y monolítica con la que se presenta al islam y a cada uno de los musulmanes, obviando su diversidad. Véase: «Ayer dos moritos le rompieron la mandíbula a mi sobrino y luego le quitaron el móvil [...]. Sí, me están haciendo muy racista estos inmigrantes», "La que lapida mujeres, ahorca homosexuales, practica la ablación, permite el matrimonio de niñas. [...]» e "¿Islamofobia? Hay que evitar la invasión del islam que fomentan los progres [...]».

Además, se identificó la superioridad o supremacía occidental enfrentada gratuitamente al islam como rasgo de las publicaciones. El denominado Occidente se sitúa automáticamente en el exponente único válido de los derechos humanos, y todas las demás culturas estarían supeditadas a ese etnocentrismo decimonónico y alienante. Estaríamos en perpetua pugna entre un creado Oriente y un recreado Occidente, que están enfrentados y que sin 
prueba alguna se da por hecho que prevalece el éxito de Occidente como desarrollo social universal, sin tener presentes las diferentes posiciones del individuo musulmán.

Con estas generalizaciones se les supone incapaces de razonar por sí mismos para decidir cómo vivir su ciudadanía, obviando miles de ejemplos existentes. Véase: «[...] «me tapo así porque soy libre. Éxito asegurado, sobre todo en los países de mayoría musulmana», "El velo no es una gorra ni un piercing, es una forma de opresión a las mujeres por más que se intente disfrazar», "[...] La democracia occidental no es perfecta, pero consagra los derechos humanos, la igualdad de todos ante la ley y el voto universal», "Muy sencillo: en mi cultura se respetan los derechos humanos, y se lucha porque todas las personas, independientemente de su sexo, idioma, etnia, opiniones..., todas las personas tengan los mismos derechos, obligaciones y oportunidades. La libertad es eso, lo demás son cuentos».

El tercer indicador explícito es la vinculación entre islam y machismo. Según los testimonios colectados, el islam y cada uno de los musulmanes, por el mero hecho de serlo, son machistas, tanto hombres como mujeres, ya que hacen derivar sumisión, supeditación de las mujeres a los hombres directamente de la citada visión monolítica negativa del supuesto islam. Véase: «[...] es opresión siempre y sometimiento [...]», «[...] si una mujer va vestida sin velo son encarceladas, retenidas y vilipendiadas hasta por las mujeres $[. .$.$] »,$ «[...] Europa está en peligro, lo de UK es abominable. CIENTOS DE MILES DE NIÑAS VIOLADAS y medios, políticos y funcionarios implicados $[\ldots] »$.

A saber, el islam es calificado en esta muestra como: negativo, generalizado, monolítico, inferior, primitivo, machista, decimonónico, anacrónico, conflictivo, anulador de libertades individuales, rígido, antepuesto a la democracia e incompatible con los derechos humanos.

De este análisis cualitativo también se deriva que no hay un prototipo o patrón único común o susceptible de establecer categorías o tipologías en cuanto a los testimonios emitidos como reacción a la campańa. Otra cualidad destacable es que los distintos imaginarios que operan en estos tuits son el reflejo directo de la propia sociedad, ya que los hemos contrastado con las estadísticas de la islamofobia en los medios del Observatorio y son una dinámica, no una reacción directa o unidireccional y aislada a la campaña concreta emitida.

Entre los hilos no hubo diferenciaciones en las cualidades discursivas expresadas de tal manera que prevaleció el discurso negativo generalizado en torno al islam, lo islámico y los musulmanes, sin presencia de los testimonios de mujeres musulmanas o de los datos académicos que atestiguaban la diversidad de situaciones y personas dentro del islam. Sin embargo, cabe advertir que, en la acción relativa al seguimiento y al análisis de los comentarios derivados del lanzamiento en Twitter de un hashtag convertido en trending topic, se detectó la presencia de mensajes que seguían estrategias evidentes en relación con la islamofobia, es decir, presentaban un claro posicionamiento 
orquestado lanzado por emisores que, de forma recurrente y a veces también manifiesta, en sus propios perfiles de Twitter se definían como detractores del islam, concibiéndolo como algo negativo socialmente. Sirva de ejemplo la imagen de la biografía que aparece en el perfil de @reaccionarioesp, en la que se define como "varón, blanco, heterosexual, de derechas. Igualdad, sí. Feminazismo, no. Estado de derecho, sí. Estado social, no. \#StopCristianofobia \#StopIslam \#StopSánchez».

Figura 2. Captura de la biografía en Twitter de @reaccionarioesp

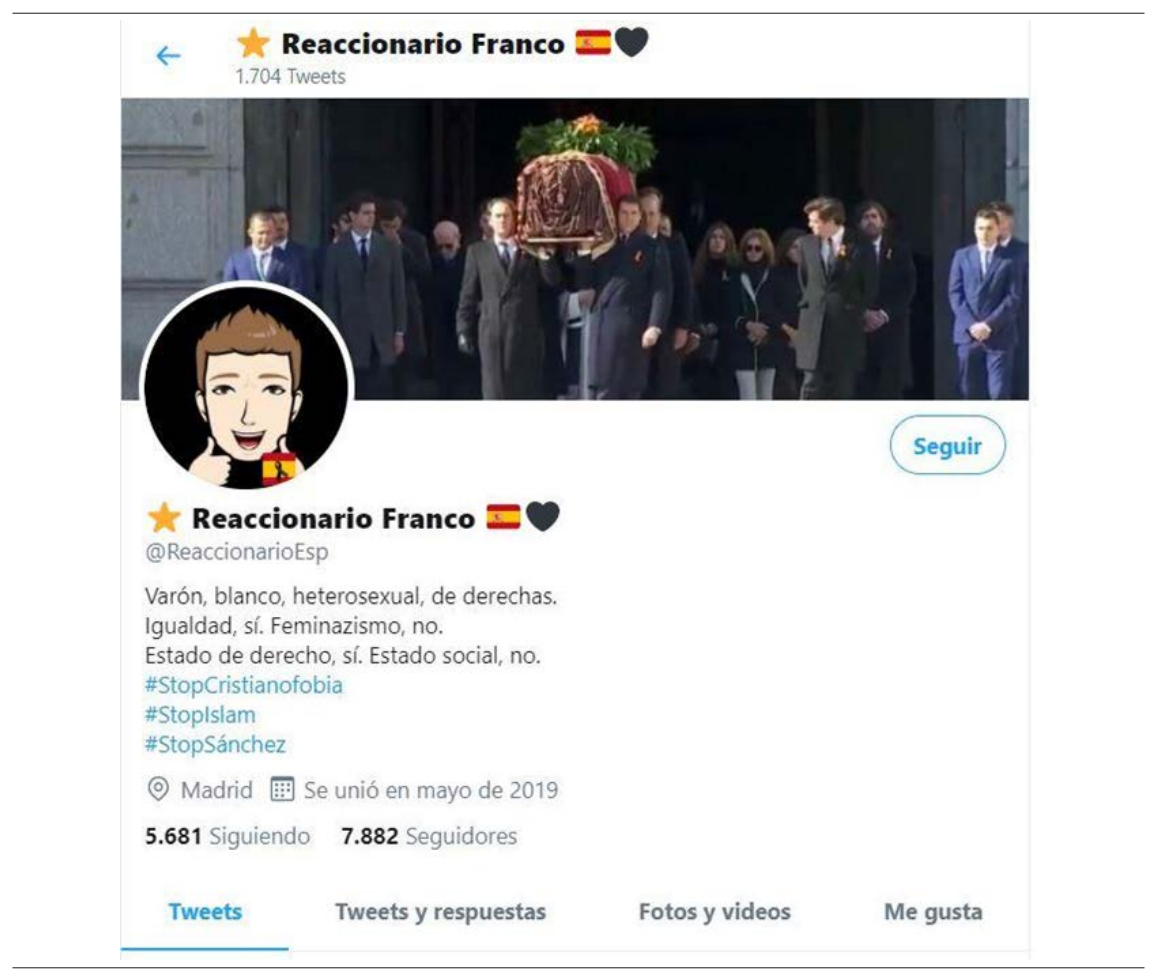

Fuente: <https://twitter.com/ReaccionarioEsp >.

\section{Conclusiones}

El análisis empírico de la campaña «Quítale las etiquetas al velo» en Twitter, con sus diferentes acciones estratégicas, nos ha permitido extraer algunas conclusiones relevantes. En líneas generales, se aprecia la espontaneidad de una conversación social que refleja el sentir de una parte de la sociedad, que se posiciona al respecto de sus actitudes hacia el islam.

La perspectiva desde donde se escriben la mayoría de los mensajes está basada en un posicionamiento que defiende una superioridad occidental desde la que se califica al islam monolíticamente de manera despectiva, una 
forma de pensar arraigada en el orientalismo y el neocolonialismo muy presente en el discurso público occidental, tanto político como mediático.

Cabe advertir que la campaña resultó exitosa en cuanto a difusión y participación, teniendo en cuenta que las reacciones positivas (en cuanto a número de retuits, mensajes positivos y "me gusta») de la primera acción de la campaña duplicaron (un $0,2 \%$ ) a las reacciones negativas (un 0,1\%). Sin embargo, el perfil de los comentarios fue mayoritariamente negativo (un $73 \%$ ), a pesar de las acciones estratégicas posteriores basadas en testimonios directos de personas musulmanas, así como testimonios de expertos académicos, que pretendían neutralizar dicha adversidad. Este dato evidencia un claro posicionamiento de los usuarios participantes a través de la expresión de actitudes negativas hacia el islam.

El análisis del perfil de dichos usuarios observado en las tres primeras acciones indica que estamos ante usuarios que actúan de forma puntual y no coordinada, emitiendo opiniones individuales desvinculadas de referentes islamófobos, excepto en algún caso aislado. Entre otras razones, los mecanismos que favorecen las interacciones sociales, así como la viralidad de los mensajes (tales como uso de hashtags, menciones, emoticonos, contenidos audiovisuales, etc.) no son utilizados por estos usuarios para favorecer la discusión pública en torno a estos temas.

Sin embargo, en el caso del HT que se promocionó a TT por Twitter, quizá debido a su mayor repercusión, es notable la presencia de usuarios que participan en la conversación con el fin de difundir cuestiones de tipo ideológico. Concretamente, la difusión es notablemente superior entre los usuarios que se posicionan en contra de la campaña. Este dato constituye una muestra de las formas de comportamiento de Twitter en conjunción con las peculiaridades de un TT promocionado: los usuarios en contra de ese HT participan mucho más que los que están a favor.

\section{Discusión}

Esta investigación ha servido para ilustrar el carácter general de las redes como polarizadoras y potenciadoras del discurso de odio. A partir de un estudio empírico basado en el seguimiento de la conversación social a raíz de una campańa específica para afrontar las actitudes islamófobas en el entorno de Twitter, se demuestra que la islamofobia es un fenómeno trasversal ideológicamente. La presencia del discurso del odio a través de actitudes islamófobas va más allá de posicionamientos ideológicos. Partiendo de la definición que de sí mismos realizan los usuarios que participan en las conversaciones analizadas se comprueba que, si bien la mayoría de la difusión se enmarca en perfiles de derechas, también existe un importante número de participantes que se definen públicamente como de izquierdas y que se muestran reaccionarios al uso del velo, con los mismos argumentos que sus antagonistas ideológicos.

Además, en consonancia con los trabajos previos apuntados, se evidencia que la capacidad de alcanzar una verdadera conversación social sobre las 
cuestiones más polemizadas del islam sigue siendo un reto entre los usuarios de Twitter. Tan solo un escaso porcentaje de los mensajes analizados tenían la intención de difundir información por parte del usuario, cifra que demuestra la escasa intención de favorecer un entendimiento sobre las cuestiones más polémicas del islam, de tal modo que nuestros resultados se suman a los numerosos estudios realizados en España que señalan cómo el discurso específico sobre la comunidad musulmana en Internet es superficial y tendencioso y está claramente estigmatizado, estableciendo constantemente una diferenciación entre un «ellos» (la comunidad musulmana) opuesto a un «nosotros».

Se valida así nuestra hipótesis y se comprueba que, lejos de alcanzar el objetivo de conseguir un verdadero diálogo social y lograr un enmarcado más constructivo de este colectivo, la campaña lanzada en Twitter solo sirvió para perpetuar la conflictividad y la polémica sustentada en las diferencias ideológicas de los usuarios.

\section{Referencias bibliográficas}

AlCÁNTARA-Plá, M. y RUIZ-SÁnCHEZ, A. (2017). «The framing of Muslims of the Spanish Internet». Lodz Papers in Pragmatics, 13 (2). $<$ https://doi.org/10.1515/lpp-2017-0013>

Arcila, C.; Blanco-Herrero D. y Valdez Apolo, M.B. (2020). «Rechazo y discurso de odio en Twitter: Análisis de contenido de los tuits sobre migrantes y refugiados en español». Reis: Revista Española de Investigaciones Sociológicas, 172, 21-40.

BANKS, J. (2010). «Regulating hate speech online». International Review of Law, Computers \& Technology, 24 (3), 233-239.

CAMPOS ZAMORA, F. (2017). «Existe un derecho a blasfemar?: Sobre libertad de expresión y discurso del odio". DOXA: Cuadernos de Filosofía del Derecho, 41, 281-295. <https://doi.org/10.14198/DOXA2018.41.14>

ChaKRABORTI, N.; GARLAND, J. y HARDY, S.J. (2014). The Leicester Hate Crime Project: Findings and Conclusions. Leicester: University of Leicester.

CHENG, J.E. (2015). «Islamophobia, Muslimophobia or racism?: Parliamentary discourses on Islam and Muslims in debates on the minaret ban in Switzerland». Discourse \& Society, 26 (5), 562-586.

Congosto, M.L. (2016). Caracterización de usuarios y propagación de mensajes en Twitter en el entorno de temas sociales. Madrid: Universidad Complutense de Madrid. Tesis doctoral.

EUROPEAN COMMISSION (2016). Code of conduct on countering illegal hate speech onli$n e$. Recuperado de <https://ec.europa.eu/info/policies/justice-and-fundamentalrights/combatting-discrimination/racism-and-xenophobia/eu-code-conductcountering-illegal-hate-speech-online_en>.

EUROPEAN COMMISSION AGAINST RACISM AND INTOLERANCE (ECRI). COUNCIL OF EUROPE (2016). Recomendación General n. ${ }^{\circ} 15$ relativa a la lucha contra el discurso de odio y su Memorándum explicativo. Estrasburgo: Comisión Europea Contra el Racismo y la Intolerancia (ECRI). Consejo de Europa. 
EVolvi, G. (2019). «\#Islamexit: inter-group antagonism on Twitter». Information, Communication \& Society, 22 (3), 386-401. <https://doi.org/10.1080/1369118X.2017.1388427>

FernándeZ, J.; GutiérreZ, Y.; GómeZ, J.M. y MArtíneZ, P. (2015). «Social Rankings: Análisis visual de sentimientos en redes sociales». Procesamiento del Lenguaje Natural, 55 (septiembre), 199-202. Recuperado de <http://journal. sepln.org/sepln/ojs/ojs/index.php/pln/article/view/5238>.

Gagliardone, I. et al. (2015). "Countering Online Hate Speech». Programme in Comparative Media Law and Policy. Oxford: University of Oxford.

GARRIDO Clemente, P. (2013). «Mujeres musulmanas y feministas». En: Trabajo social con comunidades y mujeres musulmanas: Diagnóstico de las discriminaciones múltiples. Madrid: Ediciones Académicas.

GiUliA, Evoli (2018). "Hate in a Tweet: Exploring Internet-Based Islamophobic Discourses». Religions, 9 (10), 307. $<$ https://doi.org/10.3390/rel9100307>

IEMED y FUNDACIÓN AL FANAR (ed.) (2018). Una realidad incontestable: Islamofobia en los medios. Barcelona. Recuperado de <https:/www.iemed.org/publication/ una-realidad-incontestable-islamofobia-en-los-medios/ $>$.

IGANSKI. P. y SWEIRY. A. (2018). Stopping hate: How to counter hate speech on Twitter. Media Diversity Institute (MDI). Recuperado de <https://www. media-diversity.org/resources/stopping-hate-how-to-counter-hate-speech-ontwitter/>.

KOPYTOWSKA, M. (2017). "Discourses of hate and radicalism in action». En: KOPYTOWSKA, M. (ed.). Contemporary Discourses of Hate and Radicalism across Space and Genres. Ámsterdam: John Benjamins.

MagAllón, R. (2020). Desinformación, minorias religiosas y discurso del odio. Maldita.es. Recuperado de <https://migracion.maldita.es/wp-content/uploads/2020/10/ Informe_minorias_religiosas_web-3.pdf $>$.

Miró, F. (2016). «Taxonomía de la comunicación violenta y el discurso del odio en Internet». En: TAMARIT, S. (coord.). "Ciberdelincuencia y cibervictimización». IDP: Revista de Internet, Derecho y Politica, 22, 82-107. UOC.

Paz, M. A.; Montero-Díaz, J. y Moreno-Delgado, A. (2020). «Hate Speech: A Systematized Review». Sage Open, 1-12. <https://doi.org/10.1177/2158244020973022>

PÉREZ DE LA FUENTE, O. (2010). "El enfoque español sobre el lenguaje del odio». En: MARTÍNEZ, O. y DANIEL, J. (eds.). Una discusión sobre identidad, minorías y solidaridad. Madrid: Dykinson, 133-156.

Peris-Hernández, M.; Maganto-Mateo, C. y Kortabarria-Kortazar, L. (2018). «Escala de sentimientos y emociones en las redes sociales e internet (SERSI): Fiabilidad y validez». European Journal of Investigation in Health, Psychology and Education, 8 (3), 143-155.

ROJO, P. et al. (2020). Guia para medios de comunicación "Islam, personas musulmanas y periodismo». Fundación Al Fanar. Recuperado de <http://www.fundacionalfanar. org/wordpress/wp-content/uploads/2020/07/Islamyperiodismo.pdf>.

Ruiz-SÁnCHEZ, A. y AlCÁNTARA-PlÁ, M. (2018). «De los números a los marcos: La representación de los musulmanes en el discurso online». En: XIII Congreso Internacional de Lingüistica Xeral, del 13 al 15 de junio, Vigo.

«Silencio de Europa ante la condena a 55 años de cárcel de tres activistas iraníes por negarse a llevar velo». Público (26 de agosto de 2019). Recuperado de <https:// 
www.publico.es/sociedad/silencio-europa-condena-55-anos-carcel-tres-activistasiranies-negarse-llevar-velo.html>.

Torres Kumbrian, R. y GARrido Clemente, P. (2013). «Un movimiento social contra las opresiones múltiples». Revista Internacional de Trabajo Social y Ciencias Sociales Comunitarias. Madrid: UNED.

VAN DijK, T. (2001). «El análisis crítico del discurso y el pensamiento social». Atenea Digital, 1, 18-24. 
